\title{
Anti-Malassezia-Specific IgE Antibodies Production in Japanese Patients with Head and Neck Atopic Dermatitis: Relationship between the Level of Specific IgE Antibody and the Colonization Frequency of Cutaneous Malassezia Species and Clinical Severity
}

\author{
Enshi Zhang, ${ }^{1,2}$ Takafumi Tanaka, ${ }^{2}$ Mami Tajima, ${ }^{1}$ Ryoji Tsuboi, ${ }^{1}$ Hiroshi Kato, ${ }^{3}$ \\ Akemi Nishikawa, ${ }^{3}$ and Takashi Sugita ${ }^{2}$ \\ ${ }^{1}$ Department of Dermatology, Tokyo Medical University, 6-7-1 Nishishinjuku, Shinjuku, Tokyo 160-0023, Japan \\ ${ }^{2}$ Department of Microbiology, Meiji Pharmaceutical University, 2-522-1 Noshio, Kiyose, Tokyo 204-8588, Japan \\ ${ }^{3}$ Department of Immunobiology, Meiji Pharmaceutical University, 2-522-1 Noshio, Kiyose, Tokyo 204-8588, Japan \\ Correspondence should be addressed to Takashi Sugita, sugita@my-pharm.ac.jp
}

Received 2 August 2011; Revised 15 November 2011; Accepted 15 November 2011

Academic Editor: K. Blaser

Copyright () 2011 Enshi Zhang et al. This is an open access article distributed under the Creative Commons Attribution License, which permits unrestricted use, distribution, and reproduction in any medium, provided the original work is properly cited.

\begin{abstract}
Atopic dermatitis of the head and neck (HNAD) is recognized as a separate condition. Malassezia, the predominant skin microbiota fungus, is considered to exacerbate atopic dermatitis $(\mathrm{AD})$, especially HNAD. In the present study, we investigated the relationships between the levels of specific IgE antibodies, colonization frequency of eight predominant Malassezia species, and clinical severity in 61 patients with HNAD (26 mild, 24 moderate, and 11 severe cases). As clinical severity increased, the levels of specific IgE antibodies against eight Malassezia species also increased. Species diversity of the Malassezia microbiota in scale samples from patients was analyzed by nested PCR using species-specific primers. The clinical severity of HNAD was correlated with the total level of specific IgE antibodies against Malassezia species and the number of Malassezia species detected.
\end{abstract}

\section{Introduction}

Atopic dermatitis (AD), which is characterized by dermatitis with pruritus, is a chronic disease that exhibits repeated periods of remission and deterioration. This disease is caused by hypersensitivity to dry skin, a predisposing atopic factor in which IgE antibodies related to the allergy are readily produced, and various environmental factors. Numerous IgE-inducing allergens play a role in the pathogenesis of $\mathrm{AD}$. In fact, specific IgE antibodies against environmental allergens such as mites and various food allergens are detectable in the sera of patients with AD. Since the human body is covered with an enormous number of microorganisms of a plethora of types [1], some may exacerbate the symptoms of $\mathrm{AD}$. Staphylococcus aureus is an exacerbating factor in $\mathrm{AD}$, and staphylococcal superantigen-specific IgE is found in the serum of patients with $\mathrm{AD}$, but not healthy individuals $[2,3]$.
Normally, S. aureus does not colonize healthy skin. The skin $\mathrm{pH}$ of patients with $\mathrm{AD}$ is neutral while that of healthy individuals is weakly acidic, and S. aureus does not grow well on healthy skin because it prefers a neutral $\mathrm{pH}$.

With respect to skin fungi, approximately 50 species colonize the skin of patients with $\mathrm{AD}$ [4], although the predominant fungus on the skin is the lipophilic yeast Malassezia. These microorganisms colonize sebum-rich areas such as the head, face, and neck rather than the limbs or trunk because Malassezia requires lipids for growth. During the last two decades, Malassezia has been considered to be an exacerbating factor in AD because anti-Malassezia-specific IgE is present in the serum of patients with $\mathrm{AD}$, but not healthy individuals [5-7]. In addition, clinical investigations with antifungal agents (ketoconazole or itraconazole) showed improvements in $\mathrm{AD}$ symptoms, particularly atopic dermatitis of the head and neck (HNAD) [8-12]. 
The presence of anti-Malassezia IgE has been demonstrated in patients with HNAD [7, 13-15]. The detection frequency of anti-Malassezia-specific IgE in serum was higher in patients with HNAD than in those without. Bayrou et al. [7] found IgE against Malassezia antigen in 100\% of 106 patients with HANAD, but in only $28 \%$ of 25 patients who had AD without head and neck involvement. A significant correlation was also observed between the level of Malassezia-specific IgE and clinical severity criteria, as reflected by the SCORAD index.

Currently, 14 species are recognized within the genus $M a$ lassezia. Of them, both M. globosa and M. restricta were detected in all scale samples of patients with $\mathrm{AD}$ when the $\mathrm{Ma}$ lassezia microbiota was analyzed by molecular-based cultureindependent methods [16]. The colonization level of M. restricta was approximately 1.6 times greater than that of $M$. globosa [17]. M. sympodialis was the third most predominant species, with a detection rate of $58 \%$. Other species, such as M. dermatitis, M. furfur, M. obtusa, or M. slooffiae, were detected in less than $30 \%$ of the cases.

In this study, we investigated the relationships between specific IgE and the colonization frequency of the eight most predominant Malassezia species and clinical severity levels in patients with HNAD to determine the factors that were correlated with clinical severity.

\section{Methods}

2.1. Subjects. Outpatients with HNAD $(n=61)$ at Tokyo Medical University Hospital were enrolled. The study involved patients comprising 26 mild (17 men and 9 women; mean age $34.7 \pm 10.5$ years; range, $20-63$ ), 24 moderate (16 men and 8 women; mean age $33.2 \pm 9.7$ years; range, 20-64), and 11 severe ( 7 men and 4 women; mean age $32.7 \pm 10.4$ years; range 21-51) cases. AD was diagnosed according to the criteria of Hanifin and Rajka [18]. The study protocol was approved by the Institutional Review Board, and informed consent was obtained from all subjects.

2.2. Determination of Anti-Malassezia IgE Levels. Antigens from each of eight Malassezia species were prepared ( $M$. dermatitis JCM 11469, M. furfur CBS 1878, M. globosa CBS 7966, M. japonica CBS 9432, M. obtusa CBS 7876, M. restricta CBS 7877, M. sympodialis CBS 7222, and M. slooffiae CBS 7956) according to the method of Kato et al. [19]. IgE levels against these antigens were determined using the AlaSTAT microplate system (Diagnostic Products Corporation, Los Angeles, CA, USA) with slight modifications according to the method of Kato et al. [19]. Briefly, the wells of a microtiter plate were coated with $100 \mu \mathrm{L}$ of each Malassezia antigen in phosphate-buffered saline (PBS). Serum $(50 \mu \mathrm{L})$ was then added, followed by peroxidase-labeled anti-IgE antibodies. After adding TMB $\left(3,3^{\prime}, 5,5^{\prime}\right.$-tetramethylbenzidine dissolved in hydrogen peroxide) substrate solution, the absorbance at $650 \mathrm{nM}$ was measured at $1 \mathrm{~s}$ intervals for $5 \mathrm{~min}$. IgE levels (units $/ \mathrm{mL}$ ) in the samples were calculated using a standard curve, and IgE levels greater than $0.35 \mathrm{U} / \mathrm{mL}$ were defined as a positive reaction.
2.3. Analysis of Malassezia Species Diversity. Scale samples were obtained from lesions by stripping with OpSite, which is a transparent dressing (Smith \& Nephew, Hull, UK), and Malassezia DNA was extracted directly from the dressing according to the method of Sugita et al. [20]. Briefly, the collected dressing was placed in $1 \mathrm{~mL}$ of lysing solution (100 mM Tris$\mathrm{HCl}(\mathrm{pH} 8.0), 30 \mathrm{mM}$ EDTA ( $\mathrm{pH} 8.0$ ), 0.5\% sodium dodecyl sulfate) and incubated at $100^{\circ} \mathrm{C}$ for $15 \mathrm{~min}$. After deproteinization, DNA was precipitated with ethanol and Ethachinmate (Nippon Gene, Toyama, Japan). The DNA pellet was resuspended in $30 \mu \mathrm{L}$ of TE buffer $(10 \mathrm{mM}$ Tris- $\mathrm{HCl}$ ( $\mathrm{pH}$ 8.0), $1 \mathrm{mM}$ EDTA ( $\mathrm{pH} 8.0)$ ) and stored at $-20^{\circ} \mathrm{C}$ until required. The Malassezia species diversity was investigated using nested PCR with species-specific primers, following the method of Sugita et al. [20] and Morishita et al. [21]. Briefly, the Malassezia internal transcribed spacer or intergenic spacer region of the rRNA gene was amplified by PCR with Malassezia universal primers. The product of this first amplification $(1 \mu \mathrm{L})$ was used in the nested PCR step with species-specific primers.

\section{Results}

3.1. Anti-Malassezia-Specific IgE Production. Malasseziaspecific IgE antibody levels against eight species in each clinical severity group (mild, moderate, and severe) are shown in Table 1. As clinical severity increased, IgE antibody levels against all eight Malassezia species were also increased. In patients with mild $\mathrm{AD}$, the level of specific IgE against $M$. restricta was the highest $(1.13 \pm 2.50 \mathrm{U} / \mathrm{mL})$, followed by $M$. dermatitis $(0.74 \pm 1.56 \mathrm{U} / \mathrm{mL})$. Specific $\mathrm{IgE}$ for these two species was also present at high levels in patients with moderate $\mathrm{AD}(7.78 \pm 8.84 \mathrm{U} / \mathrm{mL}$ for $M$. restricta and 6.13 $\pm 8.09 \mathrm{U} / \mathrm{mL}$ for $M$. dermatitis). In severe patients, with the exceptions of M. slooffiae and M. obtusa, IgE antibody levels against the remaining six Malassezia species were each greater than $15 \mathrm{U} / \mathrm{mL}$.

The detection frequencies of specific IgE antibody are also shown in Table 1; greater than $0.35 \mathrm{U} / \mathrm{mL}$ was defined as a positive reaction. The detection frequency was also correlated with the severity of symptoms. Specific IgE antibodies against $M$. restricta and $M$. dermatitis were detected in $42.3 \%$ of mild AD cases. Specific IgE antibodies against six $\mathrm{Ma}$ lassezia species, with the exceptions of $M$. slooffiae and $M$. obtusa, were detected in greater than $70 \%$ of moderate $\mathrm{AD}$ cases. Specific IgE antibodies against all eight Malassezia species were detected in more than $80 \%$ of severe cases.

3.2. Malassezia Skin Colonization Frequency. Malassezia DNA in scale samples was detected by nested PCR using species-specific primers. The colonization frequencies of eight Malassezia species among the three clinical severity groups were similar (Table 1). Also, these frequencies were similar to those of eight Malassezia in healthy subjects. Both M. restricta and M. globosa were detected in all samples. The third most predominant species was M. sympodialis (detected in $54.5-61.5 \%)$. The remaining five species were detected in less than $40 \%$ of the cases. A total of two to seven Malassezia species were detected from each patient. The average number 
TABLE 1: Level and detection frequency of specific IgE antibody and colonization frequency of Malassezia species.

\begin{tabular}{|c|c|c|c|c|c|c|c|c|c|c|}
\hline \multirow[t]{2}{*}{ Species } & \multicolumn{3}{|c|}{ Specific IgE antibody value $(\mathrm{IU} / \mathrm{mL})^{\mathrm{a}}$} & \multicolumn{3}{|c|}{$\begin{array}{l}\text { Detection frequency (\%) of } \\
\text { specific IgE antibody }\end{array}$} & \multicolumn{4}{|c|}{$\begin{array}{c}\text { Colonization frequency (\%) of } \\
\text { Malassezia species }\end{array}$} \\
\hline & Mild & Moderate & Severe & Mild & Moderate & Severe & Mild & Moderate & Severe & $\mathrm{HS}^{\mathrm{b}}$ \\
\hline M. restricta & $1.13 \pm 2.50$ & $7.78 \pm 8.84$ & $22.74 \pm 27.52$ & 42.3 & 87.5 & 100 & 100 & 100 & 100 & 100 \\
\hline M. globosa & $0.41 \pm 0.65$ & $3.24 \pm 2.74$ & $16.99 \pm 13.24$ & 34.6 & 83.3 & 100 & 100 & 100 & 100 & 100 \\
\hline M. sympodialis & $0.37 \pm 0.45$ & $4.46 \pm 4.74$ & $20.74 \pm 16.52$ & 30.8 & 83.3 & 100 & 61.5 & 58.3 & 54.5 & 36.7 \\
\hline M. dermatitis & $0.74 \pm 1.56$ & $6.13 \pm 8.09$ & $34.47 \pm 30.89$ & 42.3 & 79.2 & 100 & 38.5 & 37.5 & 27.3 & 30.0 \\
\hline M. japonica & $0.26 \pm 0.26$ & $1.49 \pm 1.70$ & $21.85 \pm 20.82$ & 26.9 & 83.3 & 90.9 & 11.5 & 12.5 & 18.2 & 10.0 \\
\hline M. furfur & $0.28 \pm 0.36$ & $2.29 \pm 2.89$ & $11.92 \pm 12.94$ & 26.9 & 79.2 & 100 & 15.4 & 29.2 & 18.2 & 26.7 \\
\hline M. slooffiae & $0.13 \pm 0.09$ & $0.58 \pm 0.60$ & $5.55 \pm 9.57$ & 3.8 & 50.0 & 81.8 & 19.2 & 16.7 & 9.1 & 16.7 \\
\hline M. obtusa & $0.16 \pm 0.13$ & $1.15 \pm 1.35$ & $4.17 \pm 2.92$ & 11.5 & 62.5 & 90.9 & 19.2 & 16.7 & 18.2 & 10.0 \\
\hline
\end{tabular}

a, Mean + standard deviation;

b, HS: healthy subject [22].

TABLE 2: Relationship between the number of species detected and total IgE antibody levels against Malassezia species in each case.

\begin{tabular}{lccc}
\hline Severity group & \multicolumn{2}{c}{ Number of species detected and total IgE antibody levels (IU/mL) } \\
& Less than three species & Four or five species & More than six species \\
\hline Mild & $1.27+1.33(13)^{\mathrm{a}}$ & $4.33+7.00(9)$ & $8.62+6.40(4)$ \\
Moderate & $8.75+8.41(12)$ & $43.44+20.27(8)$ & $49.58+27.04(4)$ \\
Severe & $114.48+89.27(8)$ & $102.38(1)$ & $248.20+123.74(2)$ \\
\hline
\end{tabular}

${ }^{a}$ Mean + standard deviation. Number of sample examined is shown in parentheses.

of species detected in each patient was similar among the three clinical severity groups $(3.7 \pm 1.6,3.7 \pm 1.6$, and 3.5 \pm 1.4 species in mild, moderate, and severe AD cases, resp.).

The number of species detected in each severity group was compared with the total IgE antibody levels against $\mathrm{Ma}$ lassezia species. As the number of detected species increased, total level IgE antibody levels against Malassezia species also increased (Table 2).

\section{Discussion}

In this study, we investigated the relationships between specific IgE levels and the colonization frequency of the eight predominant Malassezia species and clinical severity in patients with HNAD to elucidate the factors correlated with clinical severity. Some studies have reported that the frequency of anti-Malassezia-specific IgE antibodies in serum was higher in patients with HNAD than in those without. However, the detection frequency of anti-Malassezia-specific IgE antibodies in patients with $\mathrm{HNAD}$ was different in each report: $100 \%$ [14], 68\% [23], 55\% [24], 55\% [15], and 35\% [13].

Several factors may explain this variance. Of them, the antigen used might have a significant influence on the frequency of detection. These aforementioned studies had prepared Malassezia antigens from M. furfur or M. sympodialis to detect specific IgE antibodies from patients' sera. Unfortunately, these microorganisms are not the predominant species on the skin of patients with AD. M. globosa and $M$. restricta were detected in all patients, while $M$. furfur and M. sympodialis were observed in 15.4-29.2\% and $54.5-61.5 \%$ of the patients, respectively. In addition to detection frequency, colonization levels of $M$. globosa and $M$. restricta accounted for greater than $90 \%$ of the entire Malassezia microbiota [25]. Therefore, M. globosa and $M$. restricta may play a role in exacerbating $\mathrm{AD}$. Based on this result, Kato et al. [19] quantified specific IgE against eight Malassezia species, M. dermatitis, M. furfur, M. globosa, $M$. obtusa, M. pachydermatitis, M. slooffiae, M. sympodialis, and M. restricta, in sera from patients with $\mathrm{AD}$ by enzymelinked immunosorbent assay (ELISA). The level of specific IgE antibodies for $M$. restricta was greater than that against other Malassezia species. Competitive ELISA inhibition tests revealed that $M$. restricta contained species-specific as well as shared antigens.

The colonization level of Malassezia in scale samples from patients with $\mathrm{AD}$ also differed according to clinical severity. The extent of Malassezia colonization between patients with mild and moderate AD was similar. However, colonization in severe patients was two- to fivefold higher than that of mild and moderate patients [26]. In addition, the proportions of the two predominant species, M. globosa and $M$. restricta, differed according to the clinical severity. In patients with mild and moderate $\mathrm{AD}, M$. restricta predominated over M. globosa, whereas the proportions of M. globosa and $M$. restricta were almost identical in patients with severe $\mathrm{AD}$. AD severity had no effect on Malassezia species diversity in patients with 3.45-3.71 species colonizing the skin; however, the number of species detected ranged from two to seven of a total of eight. The number of species detected in each case correlated with the total level of specific IgE antibodies against Malassezia species in each severity group (Table 2). 
Therefore, to elucidate the factors associated with the clinical severity of $\mathrm{AD}$, the relationships between specific IgE levels and colonization levels with the eight most predominant Malassezia species should be investigated.

\section{Conclusion}

The clinical severity of HNAD was correlated with total levels of IgE antibodies against Malassezia species and the number of Malassezia species detected in each case.

\section{Acknowledgments}

This study was supported in part by a research grant from the Japan Society for the Promotion of Science (to T. Sagita) and a research grant for a "High-Tech Research Center Project" from the Ministry of Education, Culture, Sports, Science and Technology, Japan (to T. Sagita).

\section{References}

[1] E. A. Grice and J. A. Segre, "The skin microbiome," Nature Reviews Microbiology, vol. 9, no. 4, pp. 244-253, 2011.

[2] I. D. Cardona, H. C. Sang, and D. Y. M. Leung, "Role of bacterial superantigens in atopic dermatitis: implications for future therapeutic strategies," American Journal of Clinical Dermatology, vol. 7, no. 5, pp. 273-279, 2006.

[3] H. Fukushima, T. Hirano, N. Shibayama et al., "The role of immune response to Staphylococcus aureus superantigens and disease severity in relation to the sensitivity to tacrolimus in atopic dermatitis," International Archives of Allergy and Immunology, vol. 141, no. 3, pp. 281-289, 2006.

[4] E. Zhang, T. Tanaka, M. Tajima, R. Tsuboi, A. Nishikawa, and T. Sugita, "Characterization of the skin fungal microbiota in patients with atopic dermatitis and in healthy subjects," Microbiology and Immunology, vol. 55, no. 9, pp. 625-632, 2011.

[5] M. W. Wessels, G. Doekes, A. G. Van Ieperen-Van Dijk, W. J. Koers, and E. Young, "IgE antibodies to Pityrosporum ovale in atopic dermatitis," British Journal of Dermatology, vol. 125, no. 3, pp. 227-232, 1991.

[6] A. Broberg, J. Faergemann, S. Johansson, S. G. O. Johansson, I. L. Strannegard, and E. Svejgaard, "Pityrosporum ovale and atopic dermatitis in children and young adults," Acta DermatoVenereologica, vol. 72, no. 3, pp. 187-192, 1992.

[7] O. Bayrou, C. Pecquet, A. Flahault, C. Artigou, N. Abuaf, and F. Leynadier, "Head and neck atopic dermatitis and Malassezia-furfur-specific IgE antibodies," Dermatology, vol. 211, no. 2, pp. 107-113, 2005.

[8] O. Back, A. Scheynius, and S. G. O. Johansson, "Ketoconazole in atopic dermatitis: therapeutic response is correlated with decrease in serum IgE," Archives of Dermatological Research, vol. 287, no. 5, pp. 448-451, 1995.

[9] A. Broberg and J. Faergemann, "Topical antimycotic treatment of atopic dermatitis in the head/neck area. A double-blind randomised study," Acta Dermato-Venereologica, vol. 75, no. 1, pp. 46-49, 1995.

[10] O. Bäck and J. Bartosik, "Systemic ketoconazole for yeast allergic patients with atopic dermatitis," Journal of the European Academy of Dermatology and Venereology, vol. 15, no. 1, pp. 34-38, 2001.
[11] E. Svejgaard, P. Ø. Larsen, M. Deleuran, T. Ternowitz, J. Roed-Petersen, and J. Nilsson, "Treatment of head and neck dermatitis comparing itraconazole $200 \mathrm{mg}$ and $400 \mathrm{mg}$ daily for 1 week with placebo," Journal of the European Academy of Dermatology and Venereology, vol. 18, no. 4, pp. 445-449, 2004.

[12] Z. Ikezawa, M. Kondo, M. Okajima, Y. Nishimura, and M. Kono, "Clinical usefulness of oral itraconazole, an antimycotic drug, for refractory atopic dermatitis," European Journal of Dermatology, vol. 14, no. 6, pp. 400-406, 2004.

[13] E. Jensen-Jarolim, L. K. Poulsen, H. With, M. Kieffer, V. Ottevanger, and P. S. Skov, "Atopic dermatitis of the face, scalp, and neck: type I reaction to the yeast Pityrosporum ovale?" Journal of Allergy and Clinical Immunology, vol. 89, no. 1 I, pp. 44-51, 1992.

[14] S. A. Devos and P. G. M. Van Der Valk, "The relevance of skin prick tests for Pityrosporum ovale in patients with head and neck dermatitis," Allergy, vol. 55, no. 11, pp. 1056-1058, 2000.

[15] C. Johansson, M. H. Sandström, J. Bartosik et al., "Atopy patch test reactions to Malassezia allergens differentiate subgroups of atopic dermatitis patients," British Journal of Dermatology, vol. 148, no. 3, pp. 479-488, 2003.

[16] T. Sugita, T. Boekhout, A. Velegraki et al., "Epidemiology of Malassezia-related skin diseases," in Malassezia and the Skin, pp. 65-120, Springer, New York, NY, USA, 2010.

[17] T. Sugita, M. Tajima, H. Tsubuku, R. Tsuboi, and A. Nishkikawa, "Quantitative analysis of cutaneous Malassezia in atopic dermatitis patients using real-time PCR," Microbiology and Immunology, vol. 50, no. 7, pp. 549-552, 2006.

[18] J. M. Hanifin and G. Rajka, "Diagnostic features of atopic dermatitis," Acta Dermato Venereologica, vol. 92, supplement 92, pp. 44-47, 1980.

[19] H. Kato, T. Sugita, Y. Ishibashi, and A. Nishikawa, "Detection and quantification of specific IgE antibodies against eight $M a$ lassezia species in sera of patients with atopic dermatitis by using an enzyme-linked immunosorbent assay," Microbiology and Immunology, vol. 50, no. 11, pp. 851-856, 2006.

[20] T. Sugita, H. Suto, T. Unno et al., "Molecular analysis of Malassezia microflora on the skin of atopic dermatitis patients and healthy subjects," Journal of Clinical Microbiology, vol. 39, no. 10, pp. 3486-3490, 2001.

[21] N. Morishita, Y. Sei, and T. Sugita, "Molecular analysis of Malassezia microflora from patients with pityriasis versicolor," Mycopathologia, vol. 161, no. 2, pp. 61-65, 2006.

[22] T. Sugita, "Molecular analysis of cutaneous microbiota and its application to the development of a new therapeutic method for microbiota-related skin diseases-Example of Malassezia and atopic dermatitis," IFO Research Communication, vol. 22, pp. 63-67, 2008.

[23] T. Y. Kim, I. G. Jang, Y. M. Park, H. O. Kim, and C. W. Kim, "Head and neck dermatitis: the role of Malassezia furfur, topical steroid use and environmental factors in its causation," Clinical and Experimental Dermatology, vol. 24, no. 3, pp. 226231, 1999.

[24] P. Mayser and A. Gross, "Ige antibodies to Malassezia furfur, M. sympodialis and Pityrosporum orbiculare in patients with atopic dermatitis, seborrheic eczema or pityriasis versicolor, and identification of respective allergens," Acta DermatoVenereologica, vol. 80, no. 5, pp. 357-361, 2000.

[25] Z. Gao, G. I. Perez-Perez, Y. Chen, and M. J. Blaser, "Quantitation of major human cutaneous bacterial and fungal populations," Journal of Clinical Microbiology, vol. 48, no. 10, pp. 3575-3581, 2010. 
[26] M. Kaga, T. Sugita, and A. Nishikawa, "Molecular analysis of the cutaneous Malassezia microbeota from the skin of patients with atopic dermatitis of different severities," Mycoses, vol. 54, no. 4, pp. e24-e28, 2011. 


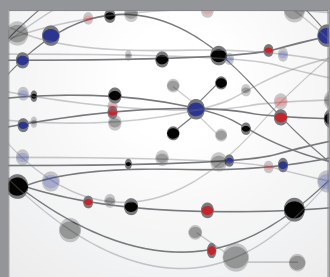

The Scientific World Journal
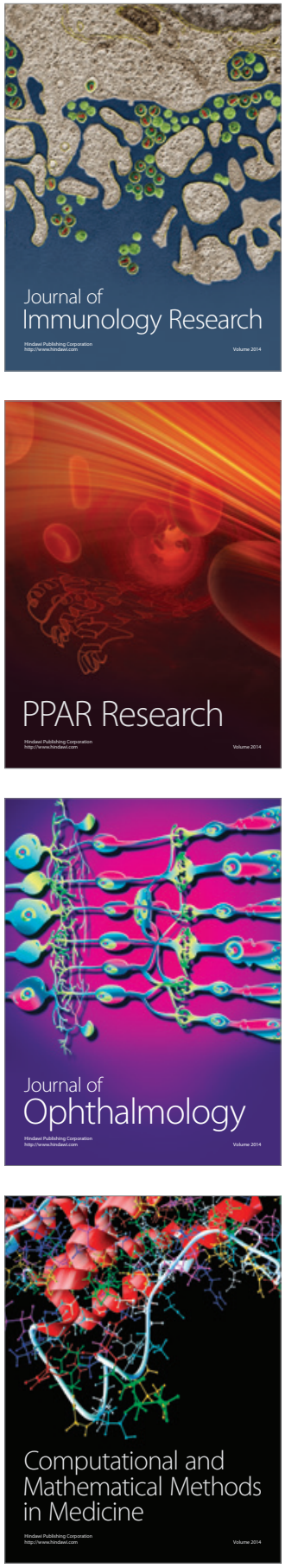

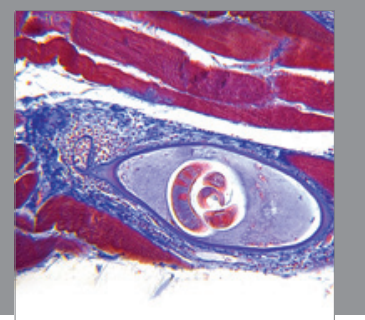

Gastroenterology

Research and Practice
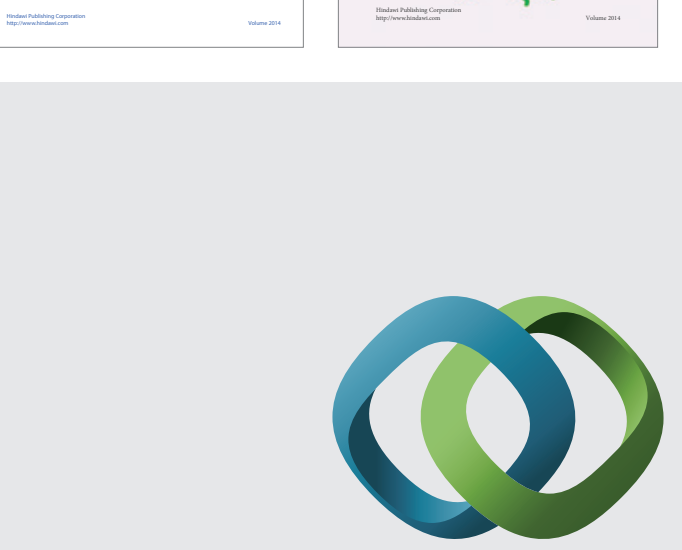

\section{Hindawi}

Submit your manuscripts at

http://www.hindawi.com
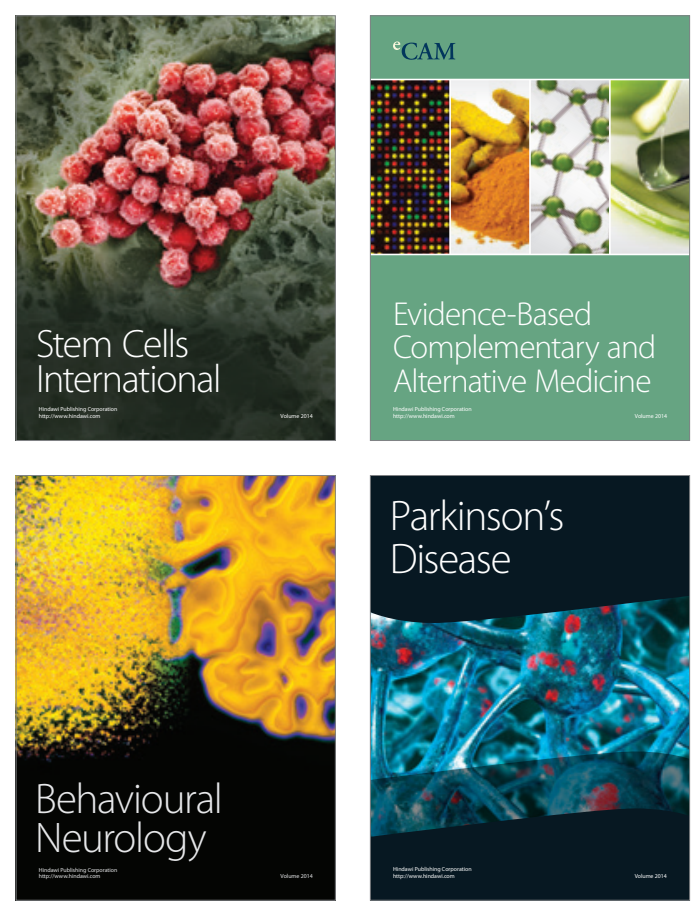

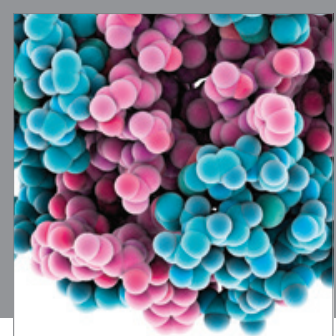

Journal of
Diabetes Research

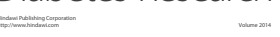

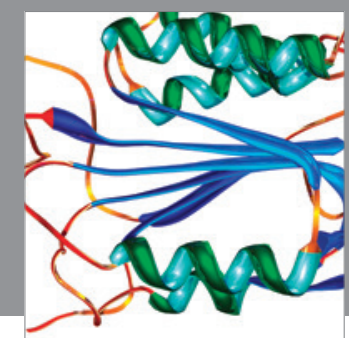

Disease Markers
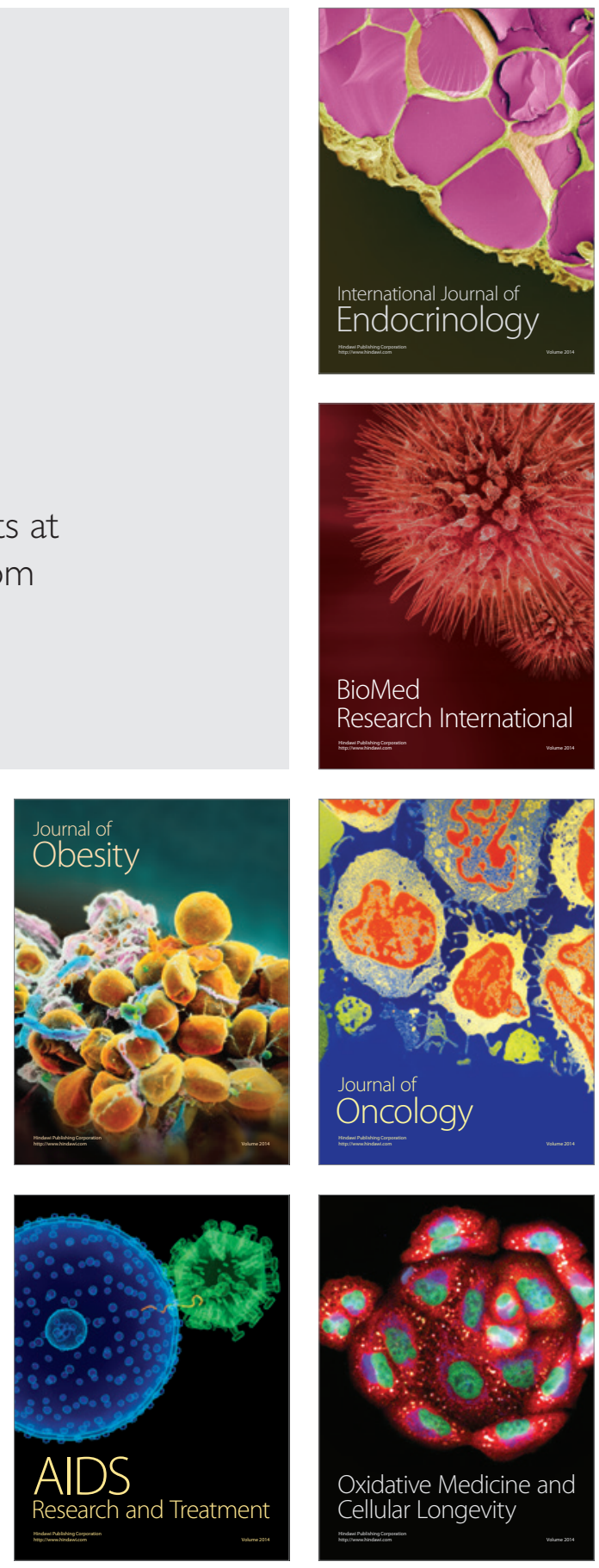